\title{
microRNA-761 regulates glycogen synthase kinase $3 \beta$ expression and promotes the proliferation and cell cycle of human gastric cancer cells
}

\author{
XINFANG SUN, HONGTAO HOU, KE LI and MINGMING ZHENG \\ Department of Gastroenterology, Huaihe Hospital (North Campus), Henan University, Kaifeng, Henan 475000, P.R. China
}

Received May 31, 2016; Accepted April 16, 2018

DOI: $10.3892 / \mathrm{ol} .2018 .9133$

\begin{abstract}
It has been well documented that aberrant expression of microRNAs (miRs) serves important roles in cancer progression. The present study investigated the roles of miR-761 on gastric cancer (GC) cell proliferation. Reverse transcription polymerase chain reaction indicated that miR-761 was frequently upregulated in GC tissues and cells. Overexpression of miR-761 promoted the cell proliferation, cell colony formation and cell cycle of GC cells. Bioinformatics analysis revealed that miR-761 might target the 3'-untranslated region of glycogen synthase kinase $3 \beta$, and was confirmed by luciferase reporter assay and western blot analysis. Taken together, the results of the present study revealed miR-761 as a tumor promoter in GC, and that it could be considered as a novel therapeutic target for patients with GC.
\end{abstract}

\section{Introduction}

Gastric cancer (GC) is the fourth most common lethal neoplasm and the second most common cause of cancer-associated mortality worldwide (1). The primary cause of GC with a poor prognosis is the GC being diagnosed at an advanced stage (2). In order to improve the outcome of GC, it is an urgent requirement to identify genetic events regulating $\mathrm{GC}$ cell proliferation.

An increasing number of studies have indicated that microRNAs (miRNAs/miRs) are a class of small, short non-coding RNAs (18-24 nucleotides) that have emerged as important post-transcriptional regulators by targeting the 3'-untranslated region (3'-UTR) of the mRNA of their target genes (3-5). It was reported that certain miRNAs act as oncogenes or tumor suppressors. miR-761 was reported to promote the progression and metastasis of non-small cell lung cancer by targeting ING4 and TIMP2 (6). miR-761 was revealed to

Correspondence to: Dr Xinfang Sun, Department of Gastroenterology, Huaihe Hospital (North Campus), Henan University, 115 Ximen Road, Kaifeng, Henan 475000, P.R. China

E-mail: xinfangsunhenan@sina.com

Key words: microRNA-761, gastric cancer, glycogen synthase kinase $3 \beta$, cell proliferation, cell cycle be upregulated and to regulate tumorigenesis in hepatocellular carcinoma by targeting Mitofusin-2 (7). The results of a study undertaken by Shi et al (8) indicated that miR-761 acted as a tumor suppressor that inhibited tumor progression by targeting MSI1 in ovarian carcinoma (8). However, the molecular mechanisms underlying miR-761 in GC remains largely unknown. The results of the present study demonstrated that miR-761 promoted GC cell proliferation via targeting the 3'-UTR of GSK $3 \beta$. The results provided novel insight into the mechanisms of GC tumor development mediated by miR-761.

\section{Materials and methods}

Clinical specimens. A total of 8 gastric carcinoma (GC) tissues [4 male and 4 female patients, age range 35-65 years (mean age, $40 \pm 2$ years)] and two normal gastric mucosal tissues [1 female (age 36) and 1 male (age 50) patients] were obtained from the Department of Gastroenterology, Huaihe Hospital (North campus), Henan University (Kaifeng, China) between 1 February 2015 and 1 October 2015. The present study was approved by the Ethics Committee of Huaihe Hospital (North campus), Henan University (Kaifeng, China). All participants provided written informed consent. Tissue samples were stored in frozen liquid nitrogen following collection.

Cell culture. Human gastric cancer SGC-7901, MGC-803, MKN-45 and AGS cell lines were provided by the American Type Culture Collection (Manassas, VA, USA), and maintained in Dulbecco's modified Eagle's medium (DMEM; Gibco; Thermo Fisher Scientific, Inc., Waltham, MA, USA) supplemented with $10 \%$ fetal bovine serum (FBS; Sigma-Aldrich; Merck KGaA, Darmstadt, Germany, USA), $100 \mathrm{U} / \mathrm{ml}$ penicillin-streptomycin (Invitrogen; Thermo Fisher Scientific, Inc.), and human gastric epithelial cells (HGECs) were purchased from Wuhan PriCells Biomedical Technology Co., Ltd. (Wuhan, China) and maintained in PriCells medium (Wuhan PriCells Biomedical Technology Co., Ltd.). All cells were cultured at $37^{\circ} \mathrm{C}$ in a humidified incubator with $5 \% \mathrm{CO}_{2}$.

Plasmids and transfection. Transfection of the cells with $2 \mu \mathrm{M}$ miRNA-761 mimics or miR-761 inhibitors (miR-761-in; GeneCopoeia, Inc., Rockville, MD, USA) and their negative controls was performed using Lipofectamine 2000 (Invitrogen; Thermo Fisher Scientific, Inc.) according to the 
manufacturer's protocols. SGC-7901 cells were infected with GSK3 $\beta$ si-RNAs, which were designed and synthesized by GeneCopoeia, Inc. Transfection of siRNAs was performed using Lipofectamine 2000, according to the manufacturer's protocols.

$R N A$ extraction and reverse transcription-quantitative polymerase chain reaction $(R T-q P C R)$. Total RNA was extracted from clinical tissues and cells using TRIzol reagent (Invitrogen; Thermo Fisher Scientific, Inc.) according to the manufacturer's protocols. The miRNA Q-PCR Detection kit (GeneCopoeia, Inc.) was used for quantification of miRNA levels according to the manufacturer's protocols. U6 was used as an internal control. The $2^{-\Delta \Delta C q}$ method was used to quantify relative RNA expression. All procedures were performed in triplicate (9).

MTT assays and colony formation. Cell proliferation assays were conducted using MTT assays, SGC-7901 cells ( $3 \times 10^{3}$ cells/well) were seeded onto 96-well plates with $100 \mu \mathrm{l}$ DMEM supplemented with 10\% FBS. Following incubation of cells for 1, 2, 3, 4, 5 and 6 days, $20 \mu \mathrm{l} 5 \mathrm{mg} / \mathrm{ml}$ MTT solution (Sigma-Aldrich; Merck KGaA) was added each well and incubated for $4 \mathrm{~h}$, and then medium was removed and $150 \mu \mathrm{l}$ DMSO (Sigma-Aldrich; Merck KGaA) was added. Next, the absorbance of each well was measured using a microplate reader set at $490 \mathrm{~nm}$.

For the colony formation assay, transfected SGC-7901 cells $\left(1 \times 10^{3}\right.$ cells/well) were added to each well of a 6 -well plate and incubated for $\sim 2$ weeks until the colony was clearly formed. Next, the cells were fixed with $4 \%$ methanol at room temperature for $30 \mathrm{~min}$ and stained with $0.5 \%$ crystal violet for $10 \mathrm{~min}$ at room temperature. Visible colonies were manually counted.

Cell cycle assays by flow cytometry. For analysis of the cell cycle, SGC-7901 cells were harvested after $48 \mathrm{~h}$ transfection, prior to being washed with PBS and then fixed in ice-cold $70 \%$ ethanol at $4^{\circ} \mathrm{C}$ overnight. The next day, the cell were incubated with RNase A at $37^{\circ} \mathrm{C}$ for $30 \mathrm{~min}$, and then stained with propidium iodide (PI; Sigma-Aldrich; Merck KGaA) at $4^{\circ} \mathrm{C}$ for $30 \mathrm{~min}$ in the dark, prior to the cells being analyzed by a flow cytometer using the CellQuest Pro software version 5.1 (BD Biosciences, Franklin Lakes, NJ, USA).

Luciferase assays. The GSK $3 \beta$ 3'-UTR and the GSK $3 \beta$ 3'-UTR mutant were amplified and cloned into the downstream of pGL3/luciferase vector (Promega Corporation, Madison, WI, USA). Cells were co-transfected with miR-761 mimics, miR-761-in or the relative miR-NC control and GSK3 $\beta$ 3'UTR or the mutant 3'UTR using Lipofectamine 2000 reagent (Invitrogen; Thermo Fisher Scientific, Inc.). Following transfection for $48 \mathrm{~h}$, firefly and Renilla luciferase activities were performed sing the dual-luciferase assay system (Promega Corporation).

Western blotting. Cells were harvested and lysed with radioimmunoprecipitation assay buffer, and the protein concentration was measured using a bicinchoninic acid assay kit (cat. no. \#23227; Thermo Fisher Scientific, Inc.). Equal amounts proteins $(50 \mu \mathrm{g})$ were separated by $10 \%$ SDS-PAGE gels and transferred onto polyvinylidene difluoride membranes. The membranes were blocked with 5\% skimmed milk at room temperature for $1 \mathrm{~h}$ and then probed with 1:1,000 diluted anti-GSK3 $\beta$ (cat. no. ab32391), anti-CyclinD1 (cat. no. ab134175) and anti-P27 (cat. no. ab32034) primary antibodies (Abcam, Cambridge, MA, USA) at $4^{\circ} \mathrm{C}$ overnight The membrane was subsequently probed with a horseradish peroxidase-conjugated secondary antibody rabbit anti-mouse IgG (cat. no. P0023D; 1:5,000; Beyotime Institute of Biotechnology, Haimen, China) for $2 \mathrm{~h}$ at room temperature. $\beta$-actin (dilution 1:5,000; cat. no. ab8227; Abcam) was used as the internal control and was incubated at $4^{\circ} \mathrm{C}$ overnight. Signals were visualized using enhanced chemiluminescent substrates (EMD Millipore, Billerica, MA, USA), according to the manufacturer's protocols, and was analyzed using the Quantity One software version 4.6 (Bio-Rad Laboratories, Inc., Hercules, CA, USA).

Statistical analysis. All data are presented as the mean \pm standard deviation and all statistical analyses were performed using SPSS 18.0 software (SPSS, Inc., Chicago, IL, USA). All experiments were repeated at least three times independently. Student's t-test was used for pair-wise comparisons and one-way analysis of variance, followed by Tukey's post hoc test was used for multiple comparisons. $\mathrm{P}<0.05$ was considered to indicate a statistically significant difference.

\section{Results}

miR-761 was upregulated in human GC tissues and GC cell lines. Initially, it was revealed that the expression levels of miR-761 were significantly upregulated in GC tissues compared with those in normal gastric mucosal tissues $(\mathrm{P}<0.05$; Fig. 1A). Similarly, significantly higher levels of miR-761 were detected in the human GC SGC-7901, MGC-803, MKN-45 and AGS cell lines compared with those in the human gastric epithelial cells (HGEC; $\mathrm{P}<0.05$; Fig. 1B). Taken together, these results indicated that miR-761 is upregulated in GC primary tumors and cell lines.

miR-761 promoted, while miR-761-in suppressed GC cell proliferation. To further characterize the functional importance of miR-761 in GC progression, its effect on the proliferation of GC cells was assessed using MTT and colony formation assays. The results of the MTT and colony formation assays indicated that overexpression of miR-761 in SGC-7901 cells significantly promoted cell proliferation and colony formation ( $\mathrm{P}<0.05$; Fig. 2A and $\mathrm{B})$. As proliferation is directly associated with the cell cycle, the effect of miR-761 on cell cycle progression was detected in SGC-7901 cells. Compared with the SGC-7901 cells transfected with miR-NC, miR-761 decreased the percentage of $\mathrm{G} 1 / \mathrm{G} 0$ phase cells, and increased the percentage of S phase cells (Fig. 2C). However, miR-761-in significantly suppressed the proliferation of GC cells using MTT and colony formation assays ( $\mathrm{P}<0.05$; Fig. $3 \mathrm{~A}$ and $\mathrm{B})$. In addition, flow cytometry revealed that miR-761-in increased the percentage of SGC-7901 cells in the G1/G0 phase, and decreased the percentage of SGC-7901 cells in the S phase (Fig. 3C). Taken together, these results suggested that miR-761 promoted GC growth by regulating the cell cycle. 

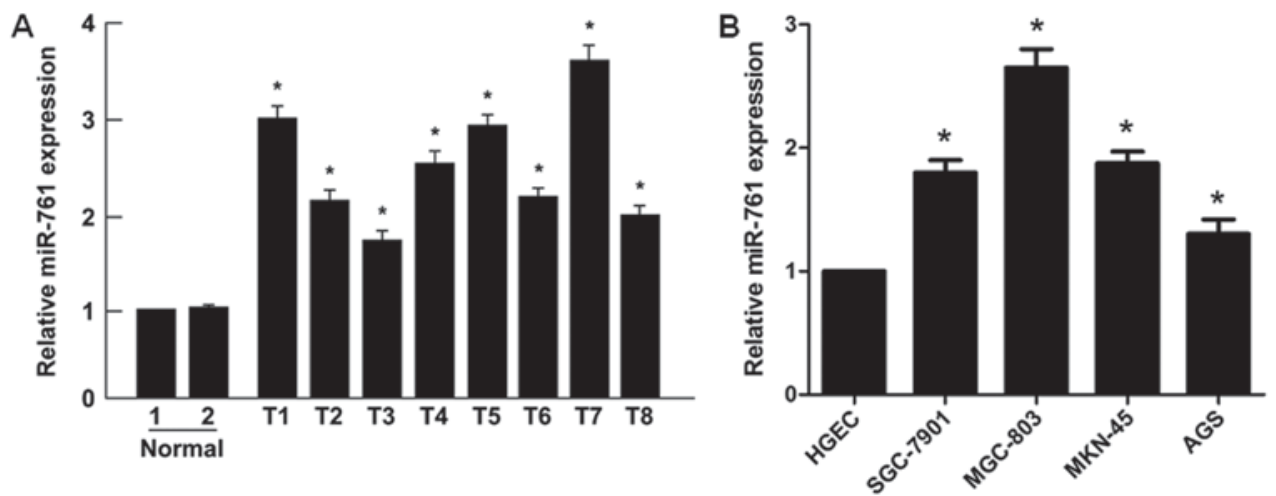

Figure 1. Upregulation of miR-761 in human GC tissues and cell lines. (A) Reverse transcription polymerase chain reaction analysis of miR-761 expression in human GC tissues and normal gastric mucosal tissues. (B) Relative expression of miR-761 in HGECs and GC SGC-7901, MGC-803, MKN-45 and AGS cell lines. Experiments were repeated $\geq 3$ times. Each bar represents the mean of three independent experiments. "P<0.05. miR, microRNA; GC, gastric cancer; HGECs, human gastric epithelial cells; GSK3 $\beta$, glycogen synthase kinase $3 \beta$.

A

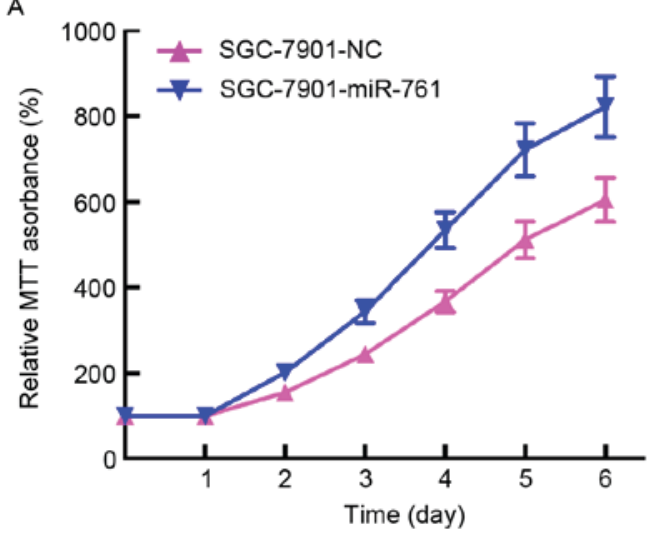

C

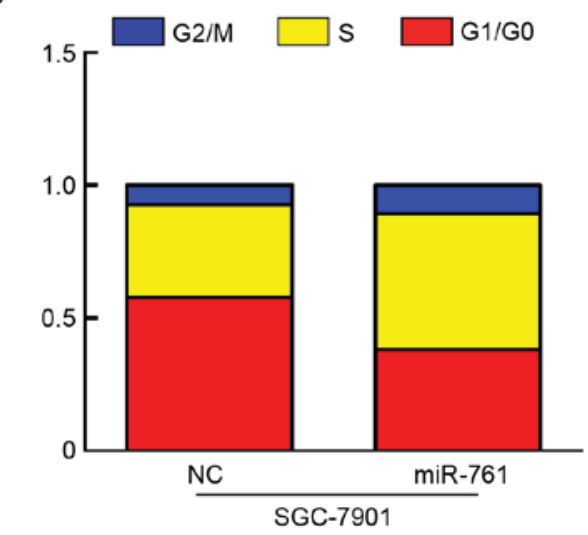

B

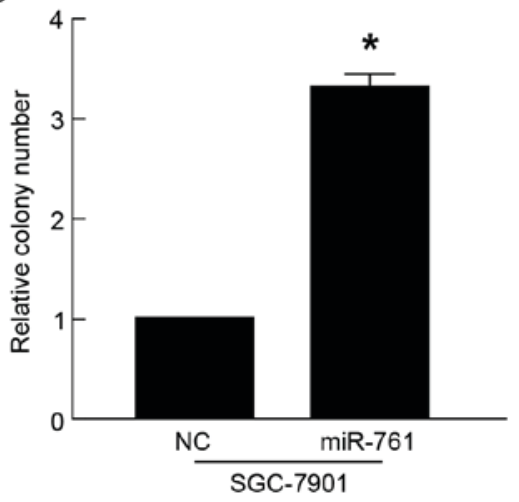

Figure 2. miR-761 upregulation promoted GC cell proliferation. (A) MTT assays revealed that upregulation of miR-761 promoted growth of the GC SGC-7901 cell line. (B) Quantification of crystal violet-stained cell colonies. (C) Flow cytometric analysis of GC SGC-7901 cells transfected with NC or miR-761. Each bar represents the mean of three independent experiments. " $\mathrm{P}<0.05$. miR, microRNA; GC, gastric cancer; NC, negative control.

miR-761 directly targets GSK3 $\beta$ by binding to its 3'-UTR and altering levels of proteins associated with cell proliferation and the cell cycle in GC cells. To investigate the molecular mechanisms by which miR-761 promoted GC cell proliferation, GSK3 $\beta$ was predicted as a potential target of miR-761 using TargetScan (http://www.targetscan.org/) (Fig. 4A). To confirm potential miR-761 binding sites in the 3'-UTR of GSK $3 \beta$, luciferase assays were used. As demonstrated in Fig. 4B, the results indicated that compared with the negative control, miR-761 attenuated the luciferase activity of the wild-type GSK3 $\beta$-3'-UTR, but had no effect on the luciferase activity of the GSK3 $\beta$-3'-UTR-mut. Furthermore, miR-761-in increased wild-type 3'-UTR GSK3 $\beta$ luciferase activity (Fig. 4C). Additionally, western blot analysis indicated that GSK3 $\beta$ expression was significantly downregulated in SGC-7901 cells following transfection with miR-761 and upregulated in miR-761-in-transfected SGC-7901 cells $(\mathrm{P}<0.05$; Fig. 4C). Cell proliferation and cell cycle regulatory 

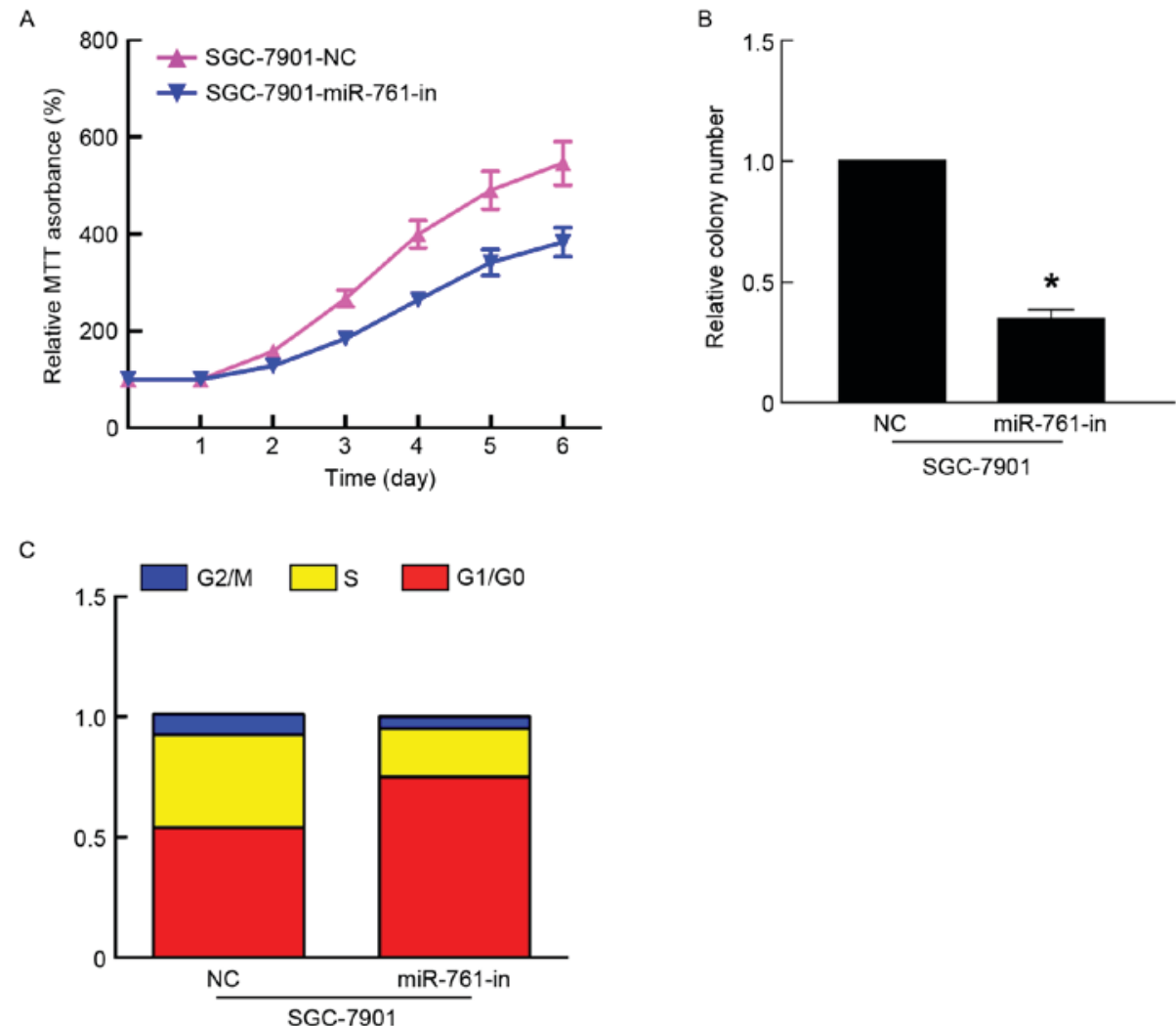

Figure 3. Inhibition of miR-761 inhibited GC cell proliferation. (A) MTT assays revealed that inhibition of miR-761 suppressed the growth of GC SGC-7901 cells. (B) Quantification of crystal violet-stained cell colonies. (C) Flow cytometric analysis of GC SGC-7901 cells transfected with NC or miR-761-in. Each bar represents the mean of three independent experiments. "P<0.05. miR, microRNA; GC, gastric cancer; NC, negative control; in, inhibitor oligonucleotides.

A
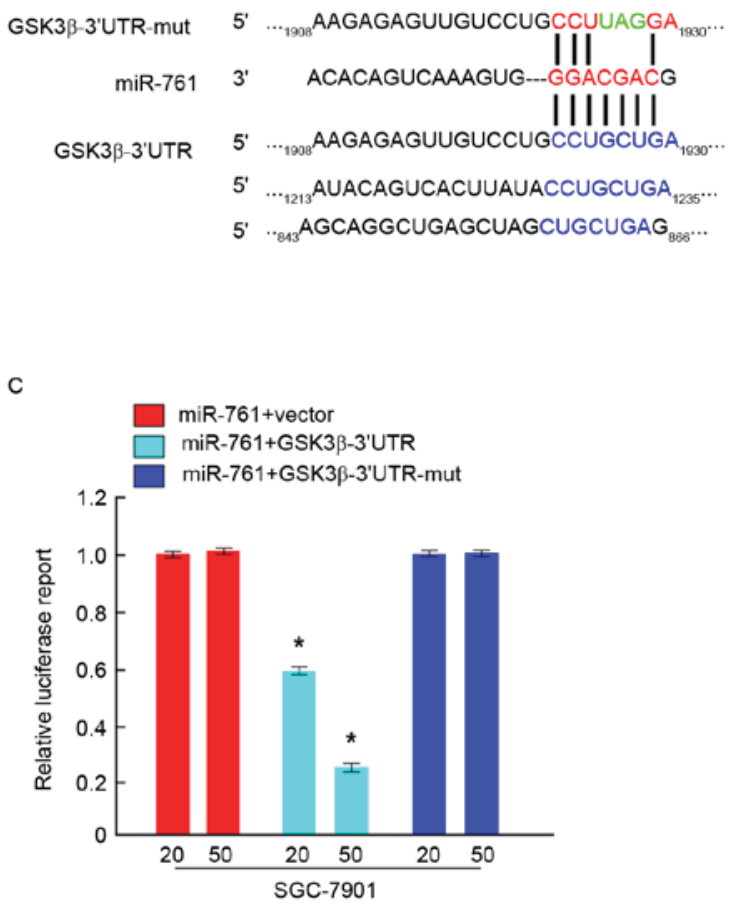

B
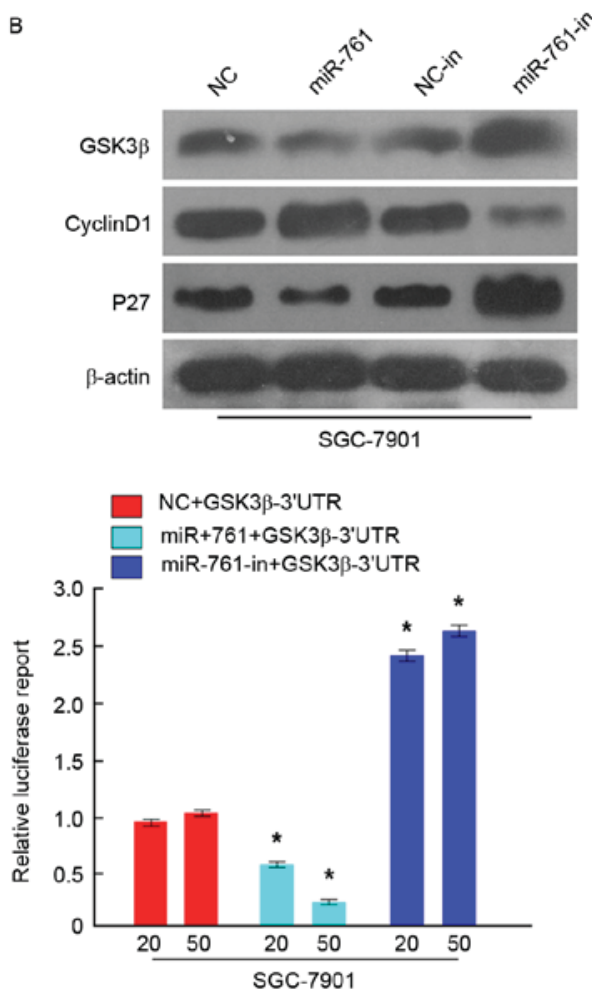

Figure 4. miR-761 suppresses GSK3 $\beta$ expression by directly targeting the GSK3 $\beta$ 3'-UTR and altered levels of proteins associated with cell proliferation and the cell cycle in GC SGC-7901 cells. (A) Predicted miR-761 target sequence in the 3'-UTR of GSK3 $\beta$ (GSK3 $\beta-3$ '-UTR) and positions of three mutated nucleotides (green) in the 3'-UTR of GSK3 $\beta$ (GSK3 $\beta$-3'-UTR mut). (B) Western blot analysis of protein expression of GSK3 $\beta$, cyclin D1 and P27 in indicated SGC-7901 cells. $\beta$-actin served as the loading control. (C) Luciferase reporter assay of SGC-7901 cells transfected with the GSK3 $\beta$-3'-UTR reporter or GSK3 $\beta$-3'-UTR-mut and miR-761 or miR-761-in. ${ }^{*} \mathrm{P}<0.05$. miR, microRNA; GSK3 $\beta$, glycogen synthase kinase $3 \beta$; UTR, untranslated region; GC, gastric cancer; mut, mutant; in, inhibitor oligonucleotides. 
genes, including Cyclin D1 and P27, were also detected by western blot analysis. The results revealed that Cyclin D1 expression was increased, while P27 expression was decreased in miR-761-overexpressing SGC-7901 cells, while miR-761-in exhibited the opposite effect (Fig. 4B).

\section{Discussion}

Numerous studies have demonstrated that miRNAs may act as activators or inhibitors of tumor proliferation (10-13). An increasing number of studies have revealed that aberrant expression of miRNAs serves essential roles in the carcinogenesis of cancer (14-16). miR-152 was reported to inhibit gastric cancer cell proliferation and motility by targeting CD151 (17). miR-935 was revealed to promote gastric cancer cell proliferation by regulating SOX7 (18). The results of a study undertaken by $\mathrm{Gu}$ et al (19) indicted that miRNA-183 suppressed apoptosis and promoted proliferation and invasion of gastric cancer cells by targeting PDCD4. miR-761 was a newly discovered oncogenic miRNA, which was upregulated and regulates tumorigenesis in hepatocellular carcinoma (7). However, the detailed biological function of miR-761 in GC remains largely unclear.

The results of the present study demonstrated that miR-761 expression levels in GC tissues and cells were significantly upregulated compared with those in normal gastric mucosal tissues and human gastric epithelial cells. Furthermore, ectopic miR-761 expression led to a lower percentage of cells in the G1/G0 phase and a higher percentage of cells in the $\mathrm{S}$ phase, and subsequently promoted GC cell proliferation and increased GC cell colony formation, while miR-761-in exhibited the opposite effect. Further experimentation was used to investigate the molecular mechanism of miR-761 in promoting cell proliferation and the cell cycle in GC. Bioinformatics analysis indicated that GSK3 $\beta$, a crucial modulator of the Wnt/ $\beta$-catenin signaling pathway (20), was the direct target of miR-761. miR-224 was reported to sustain Wnt/ $\beta$-catenin signaling and to promote the aggressive phenotype of colorectal cancer by suppressing the expression of GSK3 $\beta / S F R P 2$ (21). Deng et al (22) indicated that miR-519 promoted the progression of colorectal cancer through regulating Orai1 via regulating the Akt/GSK3 $\beta$ signaling pathway. In the present study, the results of the luciferase reporter assay and western blot analysis confirmed that miR-761 suppressed GSK $3 \beta$ expression by targeting the 3'-UTR of GSK3 $\beta$. Additionally, the cell proliferation and cell cycle regulatory genes, including Cyclin D1 and P27, were detected by western blot analysis. Cyclin D1 expression was increased, while P27 expression was decreased by miR-761 in SGC-7901 GC cells. Taken together, the results of the present study revealed that miR-761 functionally suppressed GSK3 $\beta$ expression, and subsequently regulated cellular proliferation and cell cycle regulators, Cyclin D1 and P27, which suggests that miR-761 is associated with cell proliferation and the cell cycle in GC.

In conclusion, miR-761 was upregulated and functioned as an oncogene in GC. Furthermore, it was revealed that miR-761 promoted cell proliferation and the cell cycle by targeting GSK3 $\beta$. The present study therefore demonstrated that miR-761 could be considered as a novel therapeutic target for patients with GC.

\section{Acknowledgements}

Not applicable.

\section{Funding}

No funding was received.

\section{Availability of data and materials}

All data generated or analyzed in this study are included in this article.

\section{Authors' contributions}

XS conceived and designed the experiments. XS, HH and KL performed the experiments. KL and MZ collected the samples and analyzed the data. All authors wrote and approved the final manuscript.

\section{Ethics statement and consent to participate}

The present study was approved by the Ethics Committee of Huaihe Hospital (North campus), Henan University (Kaifeng, China). All participants provided written informed consent.

\section{Patient consent for publication}

All patients provided informed consent for the publication of their data.

\section{Competing interests}

The authors declare that they have no competing interests.

\section{References}

1. Siegel R, Ma J, Zou Z and Jemal A: Cancer statistics, 2014. CA Cancer J Clin 64: 9-29, 2014.

2. Pasechnikov V, Chukov S, Fedorov E, Kikuste I and Leja M: Gastric cancer: Prevention, screening and early diagnosis. World J Gastroenterol 20: 13842-13862, 2014.

3. Shen F, Cai WS, Feng Z, Li JL, Chen JW, Cao J and Xu B: MiR-492 contributes to cell proliferation and cell cycle of human breast cancer cells by suppressing SOX7 expression. Tumour Biol 36: 1913-1921, 2015.

4. Zhang X, Ke X, Pu Q, Yuan Y, Yang W, Luo X, Jiang Q, Hu X, Gong Y, Tang K, et al: MicroRNA-410 acts as oncogene in NSCLC through downregulating SLC34A2 via activating Wnt//-catenin pathway. Oncotarget 7: 14569-14585, 2016.

5. Cheng CM, Shiah SG, Huang CC, Hsiao JR and Chang JY: Up-regulation of miR-455-5p by the TGF- $\beta$-SMAD signalling axis promotes the proliferation of oral squamous cancer cells by targeting UBE2B. J Pathol 240: 38-49, 2016.

6. Yan A, Yang C, Chen Z, Li C and Cai L: MiR-761 promotes progression and metastasis of non-small cell lung cancer by targeting ING4 and TIMP2. Cell Physiol Biochem 37: 55-66, 2015.

7. Zhou X, Zhang L, Zheng B, Yan Y, Zhang Y, Xie H, Zhou L, Zheng S and Wang W: MicroRNA-761 is upregulated in hepatocellular carcinoma and regulates tumorigenesis by targeting Mitofusin-2. Cancer Sci 107: 424-432, 2016.

8. Shi $\mathrm{C}$ and Zhang Z: miR-761 inhibits tumor progression by targeting MSI1 in ovarian carcinoma. Tumour Biol 37: 5437-5443, 2016.

9. Livak KJ and Schmittgen TD: Analysis of relative gene expression data using real-time quantitative PCR and the 2(-Delta Delta C(T)) method. Methods 25: 402-408, 2001. 
10. Yao K, He L, Gan Y,Zeng Q, Dai Y and Tan J: MiR-186 suppresses the growth and metastasis of bladder cancer by targeting NSBP1. Diagn Pathol 10: 146, 2015.

11. Wei P, Qiao B, Li Q, Han X, Zhang H, Huo Q and Sun J: MicroRNA-340 suppresses tumorigenic potential of prostate cancer cells by targeting high-mobility group nucleosome-binding domain 5. DNA Cell Biol 35: 33-43, 2016.

12. Zhang C, Liu K, Li T, Fang J, Ding Y, Sun L, Tu T, Jiang X, Du S, $\mathrm{Hu}$ J, et al: miR-21: A gene of dual regulation in breast cancer. Int J Oncol 48: 161-172, 2016.

13. Deng D, Wang L, Chen Y, Li B, Xue L, Shao N, Wang Q, Xia X, Yang Y and Zhi F: MicroRNA-124-3p regulates cell proliferation, invasion, apoptosis, and bioenergetics by targeting PIM1 in astrocytoma. Cancer Sci 107: 899-907, 2016.

14. Song H, Zhang Y, Liu N, Wan C, Zhang D, Zhao S, Kong Y and Yuan L: miR-92b regulates glioma cells proliferation, migration, invasion, and apoptosis via PTEN/Akt signaling pathway. J Physiol Biochem 72: 201-211, 2016.

15. Lu YC, Chang JT, Chan EC, Chao YK, Yeh TS, Chen JS and Cheng AJ: miR-196, an emerging cancer biomarker for digestive tract cancers. J Cancer 7: 650-655, 2016.

16. Wang F, Li B and Xie X: The roles and clinical significance of microRNAs in cervical cancer. Histol Histopathol 31: 131-139, 2016.

17. Zhai R, Kan X, Wang B, Du H, Long Y, Wu H, Tao K, Wang G Bao L, Li F and Zhang W: miR-152 suppresses gastric cancer cell proliferation and motility by targeting CD151. Tumour Biol 35: $11367-11373,2014$.
18. Yang M, Cui G, Ding M, Yang W, Liu Y, Dai D and Chen L: miR-935 promotes gastric cancer cell proliferation by targeting SOX7. Biomed Pharmacother 79: 153-158, 2016.

19. Gu W, Gao T, Shen J, Sun Y, Zheng X, Wang J, Ma J, Hu XY, Li J and Hu MJ: MicroRNA-183 inhibits apoptosis and promotes proliferation and invasion of gastric cancer cells by targeting PDCD4. Int J Clin Exp Med 7: 2519-2529, 2014.

20. Taelman VF, Dobrowolski R, Plouhinec JL, Fuentealba LC, Vorwald PP, Gumper I, Sabatini DD and De Robertis EM: Wnt signaling requires sequestration of glycogen synthase kinase 3 inside multivesicular endosomes. Cell 143: 1136-1148, 2010.

21. Li T, Lai Q, Wang S, Cai J, Xiao Z, Deng D, He L, Jiao H, Ye Y, Liang L, et al: MicroRNA-224 sustains Wnt/ $\beta$-catenin signaling and promotes aggressive phenotype of colorectal cancer. J Exp Clin Cancer Res 35: 21, 2016.

22. Deng W, Wang J, Zhang J, Cai J, Bai Z and Zhang Z: Orai1, a direct target of microRNA-519, promotes progression of colorectal cancer via Akt/GSK3 $\beta$ signaling pathway. Dig Dis Sci 61: 1553-1560, 2016.

(c) (1) (9) This work is licensed under a Creative Commons Attribution-NonCommercial-NoDerivatives 4.0 International (CC BY-NC-ND 4.0) License. 